\title{
Caspase Exploitation by Legionella pneumophila
}

\author{
Kathrin Krause and Amal O. Amer* \\ Department of Microbial Infection and Immunity, The Ohio State University, Columbus, OH, USA
}

Legionella pneumophila remains a major health concern, especially for hospitalized patients. L. pneumophila in the environment can survive extracellular or as protozoan parasite within amoeba. After human infection it efficiently replicates in alveolar macrophages without activating inflammasome assembly and cleavage of caspase- 1 . In contrast murine macrophages actively recognize intracellular $L$. pneumophila via inflammasome components which initiate pro-inflammatory cytokine secretion, phagosomal maturation and pyroptotic cell death thereby leading to bacterial restriction. During this process flagellin-dependent and -independent signaling pathways trigger the canonical as well as the non-canonical inflammasome. This review describes the current knowledge about $L$. pneumophila-induced inflammasome pathways in permissive and restrictive host cells.

Keywords: caspases, Legionella pneumophila, cell death mechanisms, phagosomal maturation, NOD like receptors

\section{OPEN ACCESS}

Edited by:

Emilio Luis Malchiodi,

University of Buenos Aires, Argentina

Reviewed by:

Eleonora Garcia Vescovi,

Instituto de Biologia Molecular y Celular de Rosario-CONICET-UNR,

Argentina

Margarita Isabel Piazzon,

Consejo Nacional de Investigaciones

Cientificas y Técnicas, Argentina

*Correspondence:

Amal O. Amer

amal.amer@osumc.edu

Specialty section:

This article was submitted to Microbial Immunology, a section of the journal

Frontiers in Microbiology

Received: 29 January 2016 Accepted: 29 March 2016 Published: 13 April 2016

Citation:

Krause K and Amer AO (2016)

Caspase Exploitation by Legionella pneumophila. Front. Microbiol. 7:515.

doi: 10.3389/fmicb.2016.00515

\section{PATHOGENESIS OF Legionella SPECIES}

Gram-negative bacteria of the genus Legionella are commonly known as the causative agent of Legionnaires' disease which account for up to $15 \%$ of community-acquired pneumonias (CAP) (Mulazimoglu and $\mathrm{Yu}, 2001$ ). Twenty species have been shown to cause infections in humans but Legionella pneumophila serogroup 1 is responsible for the majority of cases. The first outbreak of Legionnaires' disease occurred 1976 during the 56th Annual American Legion Convention which is why the subsequently identified etiologic agent was named Legionella (McDade et al., 1977). Since the clinical manifestation is an atypical pneumonia with potentially fatal outcome immediate antibiotic treatment is required. Nevertheless, the mortality rate according to the Center of Disease Control (CDC) varies from 5 to $30 \%$ with generally higher rates recorded in hospitalaquired cases (Bartram, 2007; Tai et al., 2012). The risk of disease development increases with age, preexisting conditions like chronic lung diseases, cancer and compromised immune defense after organ transplantation, glucocorticoid treatment, or chemotherapy. Although the incidence of Legionnaire's disease worldwide is unknown, with up to 18.000 annual cases in the USA, increasing numbers have been reported to the CDC in recent years. This might be due to better surveillance and diagnostic resources but also because of improved life expectancy of high-risk patients. In addition to Legionnaires' disease, Legionella species can also cause the non-pneumonic Pontiac fever. This self-limiting, influenza-like infection affects only the upper respiratory tract but is characterized by high attack rates (Tai et al., 2012).

Legionellae are globally distributed and predominantly found in natural or humanmanufactured aquatic habitats where they live as endosymbionts within free-living amoebae which provide transportation and protection against chemical disinfectants (Abu Kwaik et al., 1998; Richards et al., 2013). Infection usually occurs via inhalation of aerosols or aspiration of water from contaminated sources like showers, air conditioning systems, whirlpools, fountains, or respiratory care devices like air-humidifiers and nebulizers (Cunha et al., 2016). Within the alveolar spaces 
L. pneumophila predominantly replicates in alveolar macrophages and circulating monocytes (Horwitz and Silverstein, 1980). Upon phagocytosis, the bacteria immediately escape the endocytic pathway by avoiding phagosome-lysosomefusion (Horwitz, 1983b) and phagosomal acidification (Horwitz and Maxfield, 1984) thereby creating a niche for their survival called the Legionella-containing vacuole (LCV) (Horwitz, 1983a). Manipulation of host cell trafficking events leads to the encircling of the LCV with host organelles and Endoplasmatic Reticulum (ER) derived vesicles as well as the recruitment of vesicletransport regulating proteins like Rab1 (Tilney et al., 2001), Sec22b (Derre and Isberg, 2004), and ARF (ADP-ribosylation factor-1) (Kagan and Roy, 2002). The attachment of ER-derived vesicles to the L. pneumophila-containing phagosome is a substantial step for LCV-maturation and precedes bacterial replication (Horwitz, 1983a). Beyond that, efficient formation of a replicative compartment relies on the functionality of the Dot/Icm type IV secretion system (T4SS) (Roy et al., 1998). Legionella species possess two subtypes of T4SSs related to bacterial conjugation systems: T4ASSs like Lvh, Trb-1 and Trb-2 are not well understood and do not seem to play a role for survival in eukaryotic cells. In contrast, the highly conserved Dot/Icm T4BSS is indispensable for an intracellular life cycle and was shown to affect phagocytosis, LCV biogenesis and replication (Marra et al., 1992; Hilbi et al., 2001). Notably, allows the release of about 300 putative effector proteins with high functional redundancy between individual substrates (Burstein et al., 2009; Schroeder et al., 2010; Zhu et al., 2011; Lifshitz et al., 2013). For instance, multiple effectors have been identified which modulate the function of Rho GTPases thereby manipulating phagosomal trafficking (Hubber and Roy, 2010). Correspondingly, a $\operatorname{dot} A$ mutant strain fails to escape from the lysosomal degradation pathway, instead, its containing vacuole is characterized by the recruitment of the late endosomal marker proteins Rab7 and LAMP-1 (Roy et al., 1998). Aside from the well described Dot/Icm T4SS L. pneumophila strains express a type I (Lss) and type II (Lsp) secretion system. The T2SS, shown to execute the release of various degradative enzymes, also promotes intracellular replication in protozoan hosts and human macrophages whereas the T1SS has no documented role in host-pathogen-interactions (Jacobi and Heuner, 2003; Rossier et al., 2004). Interestingly, in contrast to human macrophages, macrophages from most imbred mouse strains are restrictive to Legionella species (Yamamoto et al., 1987, 1988). Further studies revealed that $L$. pneumophila causes efficient inflammasome assembly and caspase-1 activation in murine but not human infections (Ren et al., 2006; Santic et al., 2007; Akhter et al., 2009). Understanding of how Legionella avoids detection by innate immune defense mechanisms in permissive macrophages will be enormously relevant to control infections in the future.

\section{CASPASE-1}

The importance of caspase-1 as part of the innate immune system has been reported in the context of various types of intracellular infections like Salmonella enterica serovar typhimurium, S. flexneri, L. monocytogenes, B. pseudomallei, and L. pneumophila (Hilbi et al., 1998; Hersh et al., 1999; Tsuji et al., 2004; Sun et al., 2005). Caspases in general are intracellular cysteine proteases which induce different types of host cell death. Inflammatory caspase-1 becomes activated in multiprotein complexes, called 'inflammasomes', which assemble in response to pathogen- or danger-associated molecular patterns (PAMPs or DAMPs) within the cytosol of myeloid cells. Detection of these patterns by cytosolic NOD-like receptors (NLRs) is accompanied by conformational changes leading to oligomerization and recruitment of caspase-1. The subsequent cleavage of caspase-1 is the beginning of an inflammatory cascade with multiple outcomes including cytokine secretion, pore formation resulting in osmotic cell lysis, unconventional protein secretion, modulation of lipid biogenesis as well as gene transcription. Caspase-1 activity upon L. pneumophila infection of murine macrophages was first shown to be regulated by the NLR Naip5 (NLR family, apoptosis inhibitory protein 5) (Zamboni et al., 2006).

\section{Naip5, NIrc4, and ASC}

Although mice are generally non-permissive to L. pneumophila, macrophages from $\mathrm{A} / \mathrm{J}$ mice allow bacterial replication (Yamamoto et al., 1988; Yoshida et al., 1991) leading to the discovery that restriction is associated with the Lgn1 locus on chromosome 13 (Beckers et al., 1995). This region harbors multiple copies of Birc1/Naip genes among which Naip5 is underrepresented in the permissive $\mathrm{A} / \mathrm{J}$ mouse strain and also contains sequence differences at 14 positions (Growney and Dietrich, 2000). Accordingly the expression of Naip5, usually induced after phagocytic events, is significantly decreased in A/J macrophages (Diez et al., 2000) and transgenic restoration of Naip5 in permissive macrophages improves bactericidal activity against L. pneumophila (Diez et al., 2003; Wright et al., 2003). Caspase-1 was revealed as the responsible effector molecule for the Naip5-induced antibacterial activity since translocation of bacterial flagellin from the LCV to the host cytosol is detected by Naip5 and leads to cleavage of caspase-1 (Zamboni et al., 2006). Thus, expression of the flagellum-building subunit flagellin in combination with the Dot/Icm type 4 secretion system (T4SS) needed for perforation of the LCV membrane promotes an early caspase-1-mediated cell death leading to reduced bacterial replication and dissemination, whereas macrophages infected with non-flagellated L. pneumophila exhibit robust intracellular growth with unimpaired cell viability (Amer et al., 2006; Ren et al., 2006; Akamine et al., 2007). This type of caspase-1-induced cell death, later designated as pyroptosis, is accompanied by the release of the inflammatory cytokines Interleukin (IL) $-1 \beta$ and IL-18 which are directly processed by active caspase-1 (Figure 1).

In addition to Naip5, inflammasome assembly in response L. pneumophila has also been demonstrated to require the NLR Nlrc4/Ipaf, another sensor for cytosolic flagellin known to recruit procaspase-1 molecules via homophilic proteinprotein interactions within its CARD domain (Amer et al., 2006; Zamboni et al., 2006; Lightfield et al., 2008; Silveira and Zamboni, 2010). Consequently, Nlrc4 deletion abrogates caspase-1 processing; and bactericidal activity, pore formation as well as 


\section{Restrictive Mouse Macrophages}

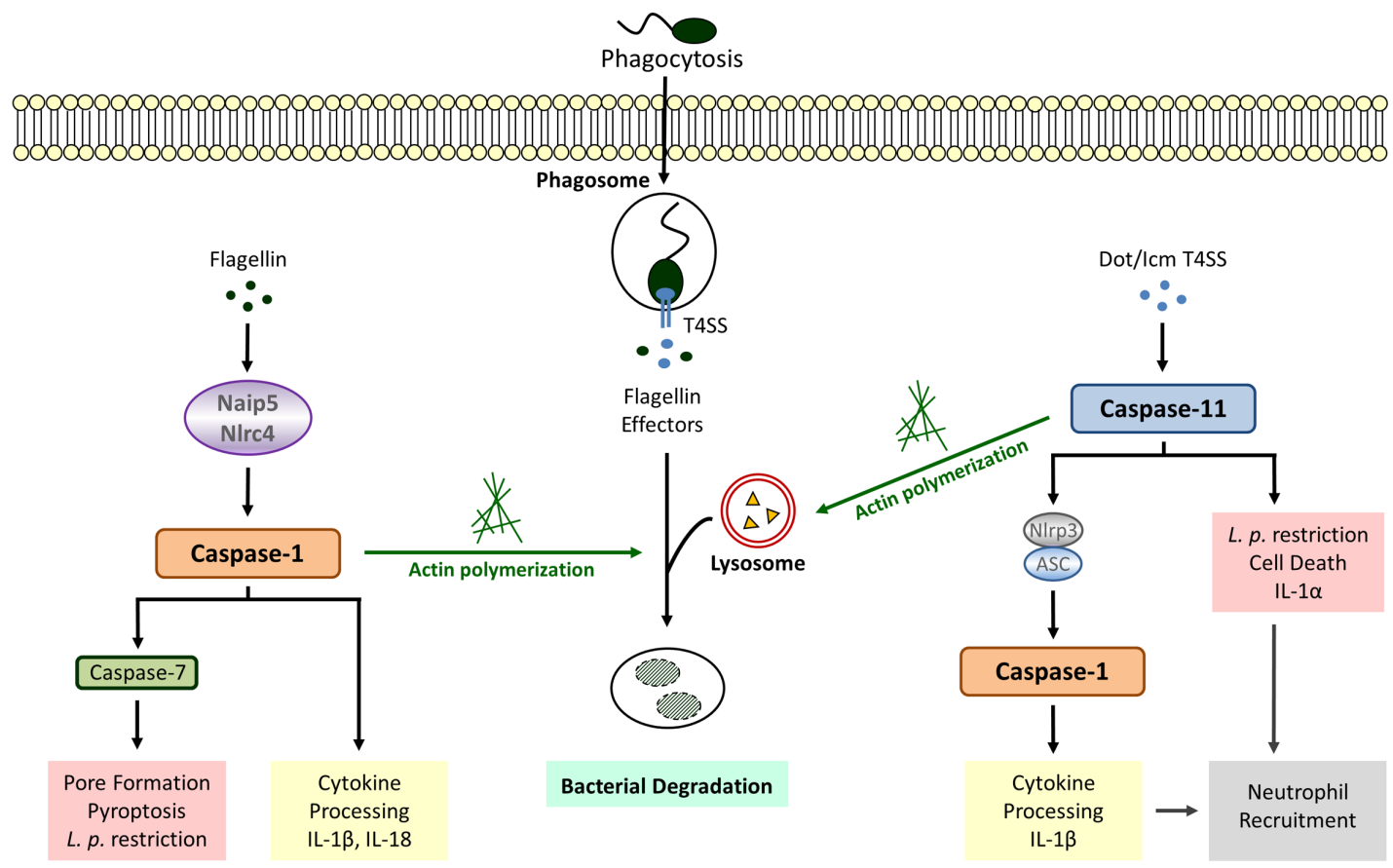

FIGURE 1 | Legionella pneumophila-induced Inflammasome Pathways in restrictive macrophages. Detection of cytosolic flagellin by Naip5/NIrc4 (canonical inflammasome) leads to cleavage of caspase-1, cytokine release, pyroptosis and bacterial growth restriction. Flagellin-independent recognition of L. pneumophila involves the activation of caspase-1 downstream of caspase-11 (non-canonical inflammasome) as well as caspase-1-independent defense strategies executed by caspase-11. Both caspase-1 and caspase-11 promote phagosome-lysosome fusion via modulation of the actin cytoskeleton.

IL-1 $\beta$ secretion. Furthermore, the Nlrc4-mediated restriction of L. pneumophila is entirely dependent on the expression of bacterial flagellin and corresponding mutants exhibit increased replication in bone marrow-derived macrophages and the lungs of infected mice (Pereira et al., 2011b). Since bacterial clearance after recognition of flagellin via Nlrc4 also applies for other non-pneumophila Legionella species it is likely to be a general mechanism of host protection against infections caused by Legionella ssp. (Silveira and Zamboni, 2010; Pereira et al., 2011a). Interestingly, human macrophages which fail to elicit an activation of caspase-1 in response to L. pneumophila infection (Figure 2) also possess significantly decreased expression levels of Nlrc4 and the adaptor protein ASC (apoptosis-associated speck-like protein) (Abdelaziz et al., 2011). Although ASC could be ruled out to play a role in Nlrc4/caspase-1-dependent growth restriction of Legionella (Zamboni et al., 2006; Pereira et al., 2011a) it was shown to function in flagellin-independent caspase1-mediated cytokine release (Zamboni et al., 2006; Case et al., 2009). Therefore, it was assumed that L. pneumophila activates two distinct caspase-1 inflammasomes in murine macrophages: one responding to flagellin that involves Naip5/Nlrc4 and leads to destruction of the host cell membrane, cell lysis, and bacterial restriction by terminating the replication cycle, and another one dependent on ASC which provokes cytokine production in a flagellin-independent manner. Moreover, ASC has been demonstrated to change the subcellular localization of Nlrc4 and caspase- 1 upon infection via recruitment to discrete puncta structures which is important not for the activation but for the cleavage of caspase-1 (Case and Roy, 2011). Even though the redistribution of Nlrc4 is unnecessary for the induction of pyroptosis, ASC takes part in restraining the extent of pore formation in order to maintain cellular homeostasis. These data support the hypothesis of two inflammasomes with distinct locations and functions. Yet, both inflammasome platforms are not completely independent and mutual interference can occur.

After the discovery of caspase-1 deficient mice being accidentally caspase-1/caspase-11 double knockout, the importance of caspase-1 for restriction of $L$. pneumophila in murine infections had to be reevaluated (Kayagaki et al., 2011). Nevertheless, new studies using single caspase-1 knockout macrophages confirmed caspase-1 as responsible molecule for Naip5/Nlrc4-mediated pyroptotic cell death and clearance of flagellated bacteria (Caution et al., 2015; Cerqueira et al., 2015).

\section{Modulation of the Cytoskeleton}

Caspase-1 activity in response to Naip5/Nlrc4 stimulation was also shown to initiate the acquisition of lysosomal instead of ER proteins to the LCV thereby increasing bacterial destruction within phagolysosomal compartments (Amer et al., 2006; Fortier et al., 2007). Recent data provide evidence that caspase-1 targets the actin-binding protein cofilin via activating the phosphatase slingshot (Caution 


\section{Permissive Human Macrophages}

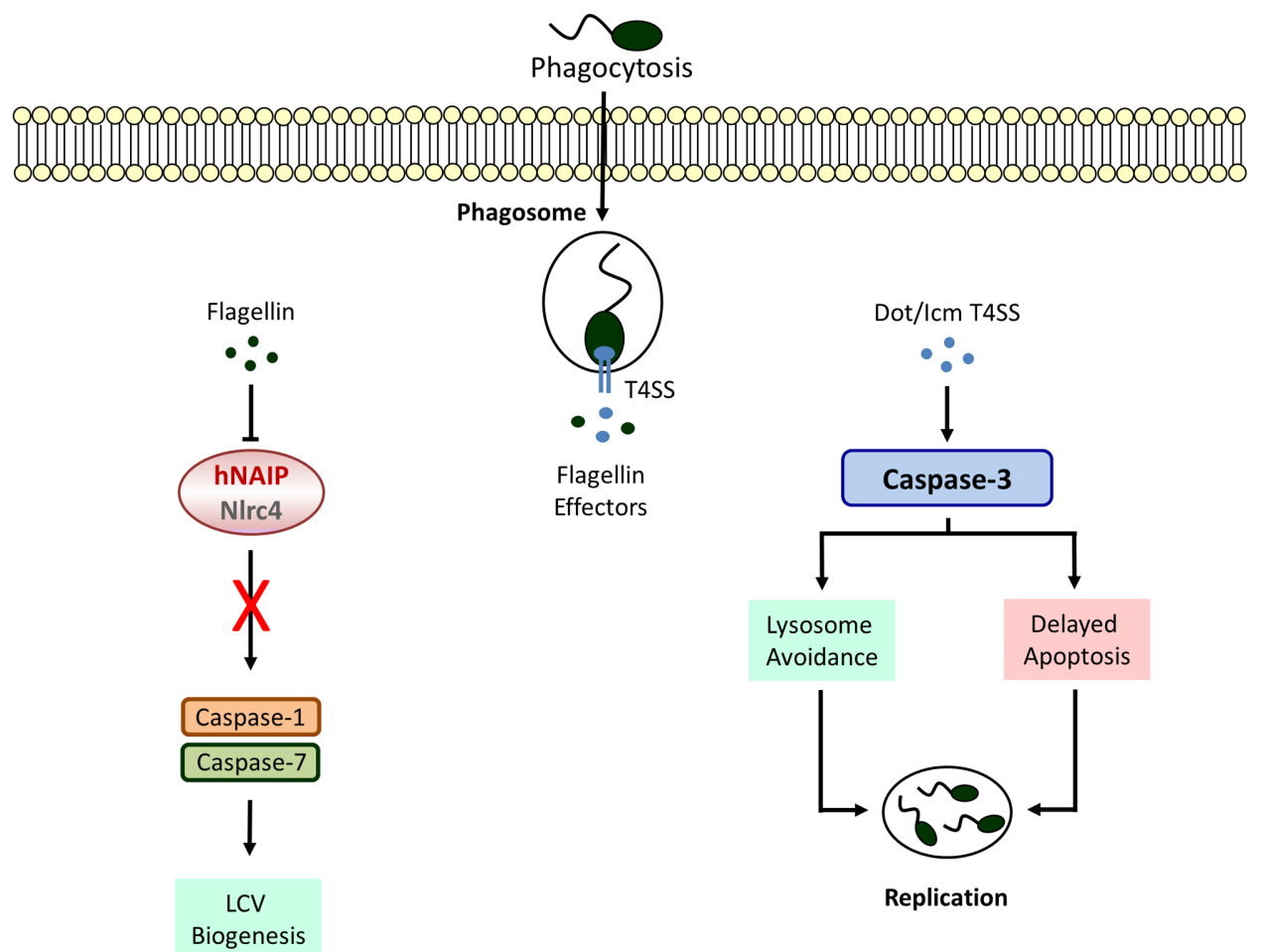

FIGURE 2 | Defective Inflammasome signaling in permissive macrophages. Human cell completely lack caspase-1 and -7 activities which allow the formation of a replicative niche called the Legionella-containing vacuole (LCV). Phagosome-lysosome fusion is avoided by the activation of caspase-3 leading to bacterial replication and egress.

et al., 2015). Dephosporylation represents the active state of cofilin allowing actin severing and polymerization in order to provide a network for vesicular trafficking. Therefore, increased lysosomal degradation of $L$. pneumophila via modulation of the cytoskeleton by caspase- 1 might be an attempt to control the intracellular infection before initiation of host cell death (Figure 1).

\section{AlM2 Inflammasome}

The recognition of flagellin by the Naip5/Nlrc4 inflammasome appears to be crucial for restriction of $L$. pneumophila, yet there is evidence that bacterial DNA leaking from the LCV can also trigger the AIM2 inflammasome leading to caspase-1 activation and pyroptosis as well (Ge et al., 2012). This process could be observed in human and murine macrophages and was significantly intensified after genetic mutation of the Dot/Icm effector protein SdhA which in the wild-type is important for maintaining the integrity of the vacuolar membrane thereby preventing a sufficient host response (Creasey and Isberg, 2012). Hence, it would be interesting to discover if pharmacological targeting of the LCV might be a way to increase caspase-1 activity in permissive macrophages due to stimulation of the AIM2 inflammasome.

\section{Negative Regulation of Inflammasome Activity}

Research so far has proven that caspase-1 is particularly important to overcome an infection with L. pneumophila. Nevertheless, it must be noted that excessive activation of caspase-1 may have detrimental implications for the host. An interesting study from von Moltke et al. (2012) describes the production of eicosanoids as consequence of Naip5/Nlrc4 inflammasome activation with L. pneumophila-derived flagellin. Local elevated eicosanoid concentrations are considered to be beneficial by the authors because they promote an influx of immune cells to the side of infection by increasing vascular permeability. In a systemic manner, however, strong eicosanoid generation leads to a substantial loss of vascular fluids, severe inflammation and rapid death. Limitation of inflammasomedependent signaling in response to $L$. pneumophila was shown to require the ubiquitin-binding protein sequestosome1 (SQSTM1/p62) also involved in autophagy (Ohtsuka et al., 2014). Caspase-1 activity as well as the secretion of IL-1 $\beta$ and IL-18 are significantly enhanced in SQSTM1 deficient macrophages infected with L. pneumophila. Additionally, L. pneumophilainduced pulmonary inflammation is more severe in the absence of SQSTM1. These results clearly indicate that even though caspase- 1 activity is indispensable for establishing a host defense 
toward Legionella species, inflammasome activation needs to be controlled to avoid an overwhelming and self-destructing immune response.

\section{CASPASE-11}

Caspase-11 takes part in the non-canonical inflammasome activation pathway in response to various gram-negative bacteria which leads to Nlrp3-dependent processing of caspase-1 (Kayagaki et al., 2011). Caspase-11 as well as the human orthologs caspase-4 and -5 directly bind to cytosolic lipopolysaccharide released from intracellular bacteria and correspondingly, LPS priming has been demonstrated to increase caspase-11 expression which is very low in resting cells (Lin et al., 2000; Hur et al., 2001; Hagar et al., 2013; Shi et al., 2014). Although the activation of caspase-11 as innate immune response against intracellular pathogens is important to control bacteria escaping from the phagosome and replicating in the cytosol of macrophages (Aachoui et al., 2013), the role of caspase-11 regarding vacuolar bacteria remains poorly defined. For instance, caspase-11 deficient mice are susceptible to L. pneumophila Lp02 (Akhter et al., 2012). Nevertheless, recent data suggest that caspase-11 is not required for restriction of opsonized L. pneumophila JR32 (Cerqueira et al., 2015). The variation in these studies might originate from the difference in strains and growth conditions used, since comparative experiments between JR32 and Lp01, the progenitor of Lp02, displayed diminished intracellular survival of Lp01 in macrophages (Samrakandi et al., 2002). In addition, Lp01 infection stimulates more phagolysosomal fusion events. It is also plausible that opsonization directs the bacterium to a caspase11-independent trafficking route, whereas bare Legionella follows a caspase-11-governed path. Thus, phenotypic difference or enhanced phagocytosis due to opsonization could very well affect the impact of caspase-11-dependent effector functions against Legionella ssp.

Although caspase-11 activation in response to L. pneumophila was shown to be Nlrc4-independent (Akhter et al., 2012), there is evidence that caspase-11 contributes to IL-1 $\beta$ secretion via triggering Nlrp3/ASC-dependent inflammasome assembly leading to caspase-1 processing (Casson et al., 2013). Interestingly, stimulation of this non-canonical pathway does not require detection of bacterial flagellin but is fully dependent on a functional Dot/Icm T4SS. Similar results could be obtained with caspase-4 in human macrophages (Casson et al., 2015). These data clearly demonstrate that there are other factors aside from flagellin which initiate a host response in L. pneumophila infected cells. Furthermore, and provided that disruption of the phagosome by bacterial secretion systems allows the translocation of bacterial components to the cytosol of the host cell, this proves that caspase-11 is activated not only in response to cytosolic but also membrane enclosed vacuolar bacteria (Figure 1).

In addition to IL-1 $\beta$ secretion, L. pneumophila-stimulated caspase-11 induction also participates in an Nlrp3/ASC- and Naip5/Nlrc4-independent release of IL- $1 \alpha$ associated with cell death and completely dependent on the presence of the T4SS
(Case et al., 2013; Casson et al., 2013). Correspondingly, downregulation of caspase-4 in L. pneumophila-infected human monocyte-derived macrophages (MDMs) significantly reduces cytotoxicity and IL-1 $\alpha$ production (Casson et al., 2015). IL- $1 \alpha$ has been demonstrated to be the primary initiator for IL-1 receptor (IL-1R)-mediated neutrophil recruitment (Barry et al., 2013) and reduction of pulmonary neutrophil infiltration revealed increased mortality during L. pneumophila infection (Tateda et al., 2001a,b). However, defective neutrophil recruitment and bacterial replication in IL- $1 \alpha$ knockout mice do not reach the same level as observed in $\mathrm{IL}-1 \mathrm{R}^{-/-}$mice indicating that IL-1 $\beta$ also plays a role (Barry et al., 2013). Another study using neutralizing antibodies confirmed a dominant role for IL-1 $\alpha$ in L. pneumophila-induced neutrophil recruitment but only depletion of both cytokines recapitulated the IL$1 \mathrm{R}^{-/-}$phenotype (Casson et al., 2013). Therefore, IL-1 $\beta$ clearly participates in the recruitment of neutrophils upon L. pneumophila infection. Given that flagellin is dispensable for both caspase-11-dependent cell death and IL- $1 \alpha / \beta$ secretion, caspase-11 activation appears to be an autonomous pathway for detection of Legionella ssp. to maintain host defense against bacteria evading the canonical inflammasome (Case et al., 2013).

Like caspase-1, caspase-11 also facilitates endocytic fusion events in response to $L$. pneumophila by modulating the actin cytoskeleton (Akhter et al., 2012). Caspase-11 deficient macrophages fail to form F-actin structures around the LCV resulting in decreased co-localization of $L$. pneumophila with lysosomal compartments. Earlier studies demonstrated that caspase-11 interacts with Aip1 (actin-interacting protein 1) in order to promote cofilin-mediated actin polymerization ( $\mathrm{Li}$ et al., 2007). With regard to intracellular L. pneumophila a physical interaction between caspase-11 and actin itself could be demonstrated in murine macrophages (Akhter et al., 2012). Furthermore, L. pneumophila-induced expression of the lipid-raft associated membrane protein flotillin-1, known to accumulate on mature phagosomes (Dermine et al., 2001), is severely diminished in caspase-11 deficient macrophages (Akhter et al., 2012). Thus, regulation of actin dynamics by caspase- 11 appears to be a crucial defense mechanism toward L. pneumophila (Figure 1).

\section{OTHER CASPASES}

Macrophages permissive to L. pneumophila do not display caspase-1 activity (Santic et al., 2007; Abdelaziz et al., 2011). Instead a strong Dot/Icm T4SS-dependent activation of caspase-3 could be demonstrated in these cells (Gao and Abu Kwaik, 1999; Zink et al., 2002). However, this does not lead to an immediate apoptotic response. In fact L. pneumophila causes rather a remarkable resistance toward cell death inducing agents at early stages of infection (Molmeret et al., 2004; AbuZant et al., 2005). The upregulation of anti-apoptotic genes due to nuclear translocation of the transcription factor NFKB is partially responsible for preventing host cell destruction (Losick and Isberg, 2006; Abu-Zant et al., 2007). Furthermore, 
SidF, a phosphatidylinositol phosphatase, blocks the apoptotic pathway by neutralizing the pro-apoptotic Bcl-2 family members BNIP3 and Bcl-rambo in U937 cells (Banga et al., 2007). The effector protein SdhA, known to protect the integrity of the LCV, is crucial for the intracellular survival of L. pneumophila and an sdhA mutant strain fails to avert host cell death (Laguna et al., 2006). Although caspase-3 has no particular role in caspase-1 competent primary murine macrophages (Zamboni et al., 2006), pre-activation of caspase3 was shown to improve bacterial clearance considerably in human U937 and murine J774 macrophage cell lines (AbuZant et al., 2005). Thus, it appears that caspase-3 activation is a distinct host response, independent of caspase-1 which is actively antagonized by intracellular L. pneumophila. This hypothesis is supported by the fact that murine dendritic cells (DCs) induce an early caspase- 3 mediated apoptotic cell death upon L. pneumophila infection to limit bacterial replication and dissemination (Nogueira et al., 2009). Interestingly and despite the functionality of the Naip5/caspase-1 pathway, pyroptosis is not needed to efficiently eradicate L. pneumophila from DCs whereas deletion of apoptotic activators like Bax and Bak results in enhanced bacterial proliferation. With regard to L. pneumophila five Dot/Icm T4SS proteins have been identified to contribute to caspase- 3 activation in DCs among which the phospholipase VipD provokes cytochrome c release via mitochondrial destabilization (Zhu et al., 2013). Accordingly, the ability to induce apoptosis is compromised in a corresponding mutant strain and accompanied by improved bacterial replication. Surprisingly, there is no reduction of L. pneumophila numbers neither in human nor in murine macrophages after loss of these proteins.

Instead of restraining intracellular replication, caspase-3 activity rather promotes growth of L. pneumophila in U937 macrophages due to cleavage of the Rab5 effector Rabaptin-5 thereby enabling evasion from the endosomal-lysosomal degradation pathway (Molmeret et al., 2004). Moreover, after pharmacological inhibition of caspase- 3 increased phagosomal maturation and trafficking to lysosomes could be observed which results in impaired bacterial survival. Yet, the importance of caspase- 3 was proven to be limited to the bacterial internalization process to subvert vesicle biogenesis whereas inhibiting caspase-3 activity at later stages of infection remains ineffective. Further studies are certainly needed to clarify why rapid stimulation of the mitochondrial apoptosis pathway is successful in DCs leading to L. pneumophila restriction but not in permissive macrophages where (regardless of active caspase-3) the apoptotic response is severely delayed and considered to serve as a tool for bacterial dissemination.

Restrictive macrophages activate caspase-1 in response to L. pneumophila but the question was raised if there are further substrates downstream of caspase-1 which take part in the Legionella-confining phenotype. Intriguingly, caspase-7, so far known to participate only in the classic apoptotic cell death pathway, was identified to be processed by caspase-1 (Lamkanfi et al., 2008). Caspase-1-dependent activation of caspase-7 in murine C57BL/6 macrophages upon infection with L. pneumophila requires detection of bacterial flagellin by the host proteins Naip5 and Nlrc4 (Akhter et al., 2009; Kofoed and Vance, 2011). Moreover, caspase-7 activity is responsible for restraining bacterial replication: firstly, because of promoting vesicle trafficking leading to bacterial degradation within phagolysosomes. Secondly, caspase-7 was shown to contribute to early apoptosis which eliminates the replicative habitat (Akhter et al., 2009). Despite the ability of permissive A/J macrophages to activate caspase-1 they lack caspase-7 activity. This might account for their incapacity to control L. pneumophila infections. Furthermore, preactivation of caspase-3 was shown to reduce bacterial loads in permissive human and murine cells but the substance staurosporine used in these experiments is a general activator of apoptosis which also affects caspase-7 (Abu-Zant et al., 2007). Fluorescent substrates measuring caspase-3 activity are known to recognize caspase-7 as well. Additional investigations are needed to clarify whether caspase-7 plays a role in human cells and if caspase-3 and -7 have distinct or overlapping functions.

\section{NOD-LIKE RECEPTORS RELATED FUNCTIONS}

The primary function of inflammasome complexes so far is considered to translate cytosolic danger signals recognized by NLR to activate downstream caspases which then initiate effector functions. Yet, there is increasing evidence that NLRs alone are capable of mediating defense mechanisms against intracellular pathogens. With regard to L. pneumophila, the fact that permissive $\mathrm{A} / \mathrm{J}$ macrophages harboring a mutant Naip5 protein display similar levels of active caspase-1 like restrictive $\mathrm{C} 57 \mathrm{BL} / 6$ macrophages in response to cytosolic flagellin suggests that Naip5 has other functions aside from merely sensing bacterial presence (Lamkanfi et al., 2007). Consistently, transgenic C57BL/6 macrophages carrying the A/J Naip5 protein allow growth of the L. pneumophila wild-type. Similar results were found for Nlrc4 deficient macrophages which display higher replication of Legionella ssp. than single caspase-1 as well as caspase-1/11 double knockout macrophages indicating the existence of a caspase-1-independent host response triggered by Nlrc4 (Pereira et al., 2011b; Cerqueira et al., 2015). Experiments with human lung epithelial cells not expressing Nlrc4 also revealed an increase in intracellular bacterial numbers after downregulation of human NAIP known to share $68 \%$ homology with murine Naip5, whereas overexpression of both exogenous Nlrc4 and endogenous NAIP improved bacterial clearance (Vinzing et al., 2008). Enhanced bacterial loads after knockdown of NAIP or Nlrc4 were also found in primary human alveolar and MDMs (Vinzing et al., 2008). Human macrophages lack caspase- 1 activity even in the presence of both molecules which emphasizes the idea of caspase-1-independent functions for NLRs. Moreover, caspase-1 is dispensable for the recruitment of Nlrc4 to subcellular puncta structures in murine macrophages (Case and Roy, 2011) and Nlrc4 also interacts with Caspase-11 (Akhter et al., 2012), thus other inflammasome platforms might exist. Overexpression of hNAIP in murine RAW264.7 
macrophages successfully promotes caspase-1 activation and bacterial growth restriction in response to flagellin-competent L. pneumophila (Katagiri et al., 2012). Contradictorily, hNAIP expressed in human-derived HEK293 cells does not respond to L. pneumophila flagellin (Zhao et al., 2011). The opposite results in human and mouse remains unclear and requires further research.

\section{UNRESOLVED ASPECTS}

Inflammasome assembly in response to L. pneumophila is very complex with multiple interaction possibilities between individual proteins. Although caspase-11 has been shown to be dispensable for the Nlrc4-mediated activation of caspase-1 (Akhter et al., 2012; Cerqueira et al., 2015), there is evidence that caspase-11 binds components of the flagellin-induced Nlrc4 inflammasome. Independently of caspase-1, caspase11 plays a role for detection of flagellin-deficient Legionella ssp. as well as IL- $1 \alpha$ secretion thereby facilitating neutrophil recruitment to the side of infection. Thus, both caspases are capable of either complementing each other or initiating completely separate defense strategies. Bacterial virulence proteins, however, or potential sensor proteins upstream of caspase-11 leading to its activation are not sufficiently clarified. Guanylate-binding proteins promote caspase-11 dependent pyroptosis in IFN- $\gamma$ stimulated macrophages due to recognition of L. pneumophila-derived cytosolic LPS (Pilla et al., 2014). Additional studies in this field may provide a better understanding of the molecular mechanism behind L. pneumophila-induced caspase-11 activation.

It is not surprising that bacteria develop mechanisms to actively suppress or evade inflammasome activation. Biofilms in fresh water structures are the main source of infection.

\section{REFERENCES}

Aachoui, Y., Leaf, I. A., Hagar, J. A., Fontana, M. F., Campos, C. G., Zak, D. E., et al. (2013). Caspase-11 protects against bacteria that escape the vacuole. Science 339, 975-978. doi: 10.1126/science.1230751

Abdelaziz, D. H., Gavrilin, M. A., Akhter, A., Caution, K., Kotrange, S., Khweek, A. A., et al. (2011). Apoptosis-associated speck-like protein (ASC) controls Legionella pneumophila infection in human monocytes. J. Biol. Chem. 286, 3203-3208. doi: 10.1074/jbc.M110.197681

Abu Khweek, A., Fernandez Davila, N. S., Caution, K., Akhter, A., Abdulrahman, B. A., Tazi, M., et al. (2013). Biofilm-derived Legionella pneumophila evades the innate immune response in macrophages. Front. Cell Infect Microbiol. 3:18. doi: 10.3389/fcimb.2013.00018

Abu Kwaik, Y., Gao, L. Y., Stone, B. J., Venkataraman, C., and Harb, O. S. (1998). Invasion of protozoa by Legionella pneumophila and its role in bacterial ecology and pathogenesis. Appl. Environ. Microbiol. 64, 3127-3133.

Abu-Zant, A., Jones, S., Asare, R., Suttles, J., Price, C., Graham, J., et al. (2007). Anti-apoptotic signalling by the Dot/Icm secretion system of L. pneumophila. Cell Microbiol. 9, 246-264. doi: 10.1111/j.1462-5822.2006.00785.x

Abu-Zant, A., Santic, M., Molmeret, M., Jones, S., Helbig, J., and Abu Kwaik, Y. (2005). Incomplete activation of macrophage apoptosis during intracellular replication of Legionella pneumophila. Infect. Immun. 73, 5339-5349. doi: 10.1128/IAI.73.9.5339-5349.2005

Akamine, M., Higa, F., Haranaga, S., Tateyama, M., Mori, N., Heuner, K., et al. (2007). Interferon-gamma reverses the evasion of Bircle/Naip5 gene
Interestingly, biofilm-derived bacteria do not express flagellin and for this reason neither initiate caspase-1/-7 activation nor pyroptosis in murine macrophages (Abu Khweek et al., 2013). Nevertheless, in human cells the absence of active caspase- 1 was proven to be flagellin-independent which raises the question whether L. pneumophila might inhibit the function of Nlrc4 with a yet unknown factor or if human Nlrc4 per se is non-responsive toward Legionella-derived flagellin.

Finally, permissive macrophages still exhibit attributes of programmed cell death but the purpose of these caspase1/11-independent pathways is controversial (Tao et al., 2013). Overall, it becomes obvious that host cell death in response to Legionella species is a delicate process which can be elicited to manage and contain intracellular infections but also provides an escape mechanism for bacterial dissemination leading to severe inflammation. Understanding how Legionella overcomes host defense mechanism in human cells will be important to develop new therapeutic strategies.

\section{AUTHOR CONTRIBUTIONS}

KK wrote the manuscript and made the figures. AA edited the manuscript and the figures.

\section{FUNDING}

Studies in AA laboratory are supported by The Cystic Fibrosis Research Grant from the Cystic Fibrosis Foundation and The Ohio State University Center for Clinical and Translational Science Longitudinal Pilot Award (CCTS). KK is supported by Deutsche Forschungsgemeinschaft (DFG - German Research Foundation).

mediated murine macrophage immunity by Legionella pneumophila mutant lacking flagellin. Microbiol. Immunol. 51, 279-287. doi: 10.1111/j.13480421.2007.tb03909.x

Akhter, A., Caution, K., Abu Khweek, A., Tazi, M., Abdulrahman, B. A., Abdelaziz, D. H., et al. (2012). Caspase-11 promotes the fusion of phagosomes harboring pathogenic bacteria with lysosomes by modulating actin polymerization. Immunity 37, 35-47. doi: 10.1016/j.immuni.2012.05.001

Akhter, A., Gavrilin, M. A., Frantz, L., Washington, S., Ditty, C., Limoli, D., et al. (2009). Caspase-7 activation by the Nlrc4/Ipaf inflammasome restricts Legionella pneumophila infection. PLoS Pathog. 5:e1000361. doi: 10.1371/journal.ppat.1000361

Amer, A., Franchi, L., Kanneganti, T. D., Body-Malapel, M., Ozoren, N., Brady, G., et al. (2006). Regulation of Legionella phagosome maturation and infection through flagellin and host Ipaf. J. Biol. Chem. 281, 35217-35223. doi: 10.1074/jbc.M604933200

Banga, S., Gao, P., Shen, X., Fiscus, V., Zong, W. X., Chen, L., et al. (2007). Legionella pneumophila inhibits macrophage apoptosis by targeting pro-death members of the Bcl2 protein family. Proc. Natl. Acad. Sci. U.S.A. 104, 51215126. doi: 10.1073/pnas.0611030104

Barry, K. C., Fontana, M. F., Portman, J. L., Dugan, A. S., and Vance, R. E. (2013). IL-1alpha signaling initiates the inflammatory response to virulent Legionella pneumophila in vivo. J. Immunol. 190, 6329-6339. doi: 10.4049/jimmunol.1300100

Bartram, J. (2007). Legionella and the Prevention of Legionellosis. Geneva: World Health Organization. 
Beckers, M. C., Yoshida, S., Morgan, K., Skamene, E., and Gros, P. (1995). Natural resistance to infection with Legionella pneumophila: chromosomal localization of the Lgn1 susceptibility gene. Mamm. Genome 6, 540-545. doi: 10.1007/BF00356173

Burstein, D., Zusman, T., Degtyar, E., Viner, R., Segal, G., and Pupko, T. (2009). Genome-scale identification of Legionella pneumophila effectors using a machine learning approach. PLoS Pathog 5:e1000508. doi: 10.1371/journal.ppat.1000508

Case, C. L., Kohler, L. J., Lima, J. B., Strowig, T., De Zoete, M. R., Flavell, R. A., et al. (2013). Caspase-11 stimulates rapid flagellin-independent pyroptosis in response to Legionella pneumophila. Proc. Natl. Acad. Sci. U.S.A. 110, 18511856. doi: 10.1073/pnas. 1211521110

Case, C. L., and Roy, C. R. (2011). Asc modulates the function of NLRC4 in response to infection of macrophages by Legionella pneumophila. MBio 2:e00117. doi: 10.1128/mBio.00117-11

Case, C. L., Shin, S., and Roy, C. R. (2009). Asc and Ipaf Inflammasomes direct distinct pathways for caspase-1 activation in response to Legionella pneumophila. Infect. Immun. 77, 1981-1991. doi: 10.1128/IAI.01382-08

Casson, C. N., Copenhaver, A. M., Zwack, E. E., Nguyen, H. T., Strowig, T., Javdan, B., et al. (2013). Caspase-11 activation in response to bacterial secretion systems that access the host cytosol. PLoS Pathog. 9:e1003400. doi: 10.1371/journal.ppat.1003400

Casson, C. N., Yu, J., Reyes, V. M., Taschuk, F. O., Yadav, A., Copenhaver, A. M., et al. (2015). Human caspase-4 mediates noncanonical inflammasome activation against gram-negative bacterial pathogens. Proc. Natl. Acad. Sci. U.S.A. 112, 6688-6693. doi: 10.1073/pnas.1421699112

Caution, K., Gavrilin, M. A., Tazi, M., Kanneganti, A., Layman, D., Hoque, S., et al. (2015). Caspase-11 and caspase- 1 differentially modulate actin polymerization via RhoA and Slingshot proteins to promote bacterial clearance. Sci. Rep. 5:18479. doi: 10.1038/srep18479

Cerqueira, D. M., Pereira, M. S., Silva, A. L., Cunha, L. D., and Zamboni, D. S. (2015). Caspase-1 but Not Caspase-11 Is Required for NLRC4mediated pyroptosis and restriction of infection by flagellated legionella species in mouse macrophages and in vivo. J. Immunol. 195, 2303-2311. doi: 10.4049/jimmunol.1501223

Creasey, E. A., and Isberg, R. R. (2012). The protein SdhA maintains the integrity of the Legionella-containing vacuole. Proc. Natl. Acad. Sci. U.S.A. 109, 3481-3486. doi: 10.1073/pnas.1121286109

Cunha, B. A., Burillo, A., and Bouza, E. (2016). Legionnaires' disease. Lancet 387, 376-385. doi: 10.1016/S0140-6736(15)60078-2

Dermine, J. F., Duclos, S., Garin, J., St-Louis, F., Rea, S., Parton, R. G., et al. (2001). Flotillin-1-enriched lipid raft domains accumulate on maturing phagosomes. J. Biol. Chem. 276, 18507-18512. doi: 10.1074/jbc.M101113200

Derre, I., and Isberg, R. R. (2004). Legionella pneumophila replication vacuole formation involves rapid recruitment of proteins of the early secretory system. Infect. Immun. 72, 3048-3053. doi: 10.1128/IAI.72.5.3048-3053.2004

Diez, E., Lee, S. H., Gauthier, S., Yaraghi, Z., Tremblay, M., Vidal, S., et al. (2003). Bircle is the gene within the Lgn1 locus associated with resistance to Legionella pneumophila. Nat. Genet. 33, 55-60. doi: 10.1038/ng1065

Diez, E., Yaraghi, Z., Mackenzie, A., and Gros, P. (2000). The neuronal apoptosis inhibitory protein (Naip) is expressed in macrophages and is modulated after phagocytosis and during intracellular infection with Legionella pneumophila. J. Immunol. 164, 1470-1477. doi: 10.4049/jimmunol.164.3.1470

Fortier, A., De Chastellier, C., Balor, S., and Gros, P. (2007). Bircle/Naip5 rapidly antagonizes modulation of phagosome maturation by Legionella pneumophila. Cell Microbiol. 9, 910-923. doi: 10.1111/j.1462-5822.2006. 00839.x

Gao, L. Y., and Abu Kwaik, Y. (1999). Activation of caspase 3 during Legionella pneumophila-induced apoptosis. Infect. Immun. 67, 4886-4894.

Ge, J., Gong, Y. N., Xu, Y., and Shao, F. (2012). Preventing bacterial DNA release and absent in melanoma 2 inflammasome activation by a Legionella effector functioning in membrane trafficking. Proc. Natl. Acad. Sci. U.S.A. 109, 6193-6198. doi: 10.1073/pnas.1117490109

Growney, J. D., and Dietrich, W. F. (2000). High-resolution genetic and physical map of the Lgn1 interval in C57BL/6J implicates Naip2 or Naip5 in Legionella pneumophila pathogenesis. Genome Res. 10, 1158-1171. doi: 10.1101/gr.10.8.1158
Hagar, J. A., Powell, D. A., Aachoui, Y., Ernst, R. K., and Miao, E. A. (2013). Cytoplasmic LPS activates caspase-11: implications in TLR4-independent endotoxic shock. Science 341, 1250-1253. doi: 10.1126/science.1240988

Hersh, D., Monack, D. M., Smith, M. R., Ghori, N., Falkow, S., and Zychlinsky, A. (1999). The Salmonella invasin SipB induces macrophage apoptosis by binding to caspase-1. Proc. Natl. Acad. Sci. U.S.A. 96, 2396-2401. doi: 10.1073/pnas.96.5.2396

Hilbi, H., Moss, J. E., Hersh, D., Chen, Y., Arondel, J., Banerjee, S., et al. (1998). Shigella-induced apoptosis is dependent on caspase-1 which binds to IpaB. J. Biol. Chem. 273, 32895-32900. doi: 10.1074/jbc.273.49.32895

Hilbi, H., Segal, G., and Shuman, H. A. (2001). Icm/dot-dependent upregulation of phagocytosis by Legionella pneumophila. Mol. Microbiol. 42, 603-617. doi: 10.1046/j.1365-2958.2001.02645.x

Horwitz, M. A. (1983a). Formation of a novel phagosome by the Legionnaires' disease bacterium (Legionella pneumophila) in human monocytes. J. Exp. Med. 158, 1319-1331. doi: 10.1084/jem.158.4.1319

Horwitz, M. A. (1983b). The Legionnaires' disease bacterium (Legionella pneumophila) inhibits phagosome-lysosome fusion in human monocytes. J. Exp. Med. 158, 2108-2126.

Horwitz, M. A., and Maxfield, F. R. (1984). Legionella pneumophila inhibits acidification of its phagosome in human monocytes. J. Cell Biol. 99, 1936-1943. doi: $10.1083 /$ jcb.99.6.1936

Horwitz, M. A., and Silverstein, S. C. (1980). Legionnaires' disease bacterium (Legionella pneumophila) multiples intracellularly in human monocytes. J. Clin. Invest. 66, 441-450. doi: 10.1172/JCI109874

Hubber, A., and Roy, C. R. (2010). Modulation of host cell function by Legionella pneumophila type IV effectors. Annu. Rev. Cell Dev. Biol. 26, 261-283. doi: 10.1146/annurev-cellbio-100109-104034

Hur, J., Kim, S. Y., Kim, H., Cha, S., Lee, M. S., and Suk, K. (2001). Induction of caspase-11 by inflammatory stimuli in rat astrocytes: lipopolysaccharide induction through p38 mitogen-activated protein kinase pathway. FEBS Lett. 507, 157-162. doi: 10.1016/S0014-5793(01)02975-1

Jacobi, S., and Heuner, K. (2003). Description of a putative type I secretion system in Legionella pneumophila. Int. J. Med. Microbiol. 293, 349-358. doi: 10.1078/1438-4221-00276

Kagan, J. C., and Roy, C. R. (2002). Legionella phagosomes intercept vesicular traffic from endoplasmic reticulum exit sites. Nat. Cell Biol. 4, 945-954. doi: $10.1038 /$ ncb883

Katagiri, N., Shobuike, T., Chang, B., Kukita, A., and Miyamoto, H. (2012). The human apoptosis inhibitor NAIP induces pyroptosis in macrophages infected with Legionella pneumophila. Microbes Infect. 14, 1123-1132. doi: 10.1016/j.micinf.2012.03.006

Kayagaki, N., Warming, S., Lamkanfi, M., Vande Walle, L., Louie, S., Dong, J., et al. (2011). Non-canonical inflammasome activation targets caspase-11. Nature 479, 117-121. doi: 10.1038/nature10558

Kofoed, E. M., and Vance, R. E. (2011). Innate immune recognition of bacterial ligands by NAIPs determines inflammasome specificity. Nature 477, 592-595. doi: 10.1038/nature10394

Laguna, R. K., Creasey, E. A., Li, Z., Valtz, N., and Isberg, R. R. (2006). A Legionella pneumophila-translocated substrate that is required for growth within macrophages and protection from host cell death. Proc. Natl. Acad. Sci. U.S.A. 103, 18745-18750. doi: 10.1073/pnas.0609012103

Lamkanfi, M., Amer, A., Kanneganti, T. D., Munoz-Planillo, R., Chen, G., Vandenabeele, P., et al. (2007). The Nod-like receptor family member Naip5/Bircle restricts Legionella pneumophila growth independently of caspase-1 activation. J. Immunol. 178, 8022-8027. doi: 10.4049/jimmunol.178.12.8022

Lamkanfi, M., Kanneganti, T. D., Van Damme, P., Vanden Berghe, T. Vanoverberghe, I., Vandekerckhove, J., et al. (2008). Targeted peptidecentric proteomics reveals caspase-7 as a substrate of the caspase-1 inflammasomes. Mol. Cell. Proteomics 7, 2350-2363. doi: 10.1074/mcp.M800132-MCP200

Li, J., Brieher, W. M., Scimone, M. L., Kang, S. J., Zhu, H., Yin, H., et al. (2007). Caspase-11 regulates cell migration by promoting Aip1-Cofilinmediated actin depolymerization. Nat. Cell Biol. 9, 276-286. doi: 10.1038/ ncb1541

Lifshitz, Z., Burstein, D., Peeri, M., Zusman, T., Schwartz, K., Shuman, H. A., et al. (2013). Computational modeling and experimental validation of the Legionella 
and Coxiella virulence-related type-IVB secretion signal. Proc. Natl. Acad. Sci. U.S.A. 110, E707-E715. doi: 10.1073/pnas.1215278110

Lightfield, K. L., Persson, J., Brubaker, S. W., Witte, C. E., Von Moltke, J., Dunipace, E. A., et al. (2008). Critical function for Naip5 in inflammasome activation by a conserved carboxy-terminal domain of flagellin. Nat. Immunol. 9, 1171-1178. doi: $10.1038 /$ ni.1646

Lin, X. Y., Choi, M. S., and Porter, A. G. (2000). Expression analysis of the human caspase-1 subfamily reveals specific regulation of the CASP5 gene by lipopolysaccharide and interferon-gamma. J. Biol. Chem. 275, 39920-39926. doi: $10.1074 /$ jbc.M007255200

Losick, V. P., and Isberg, R. R. (2006). NF-kappaB translocation prevents host cell death after low-dose challenge by Legionella pneumophila. J. Exp. Med. 203, 2177-2189. doi: 10.1084/jem.20060766

Marra, A., Blander, S. J., Horwitz, M. A., and Shuman, H. A. (1992). Identification of a Legionella pneumophila locus required for intracellular multiplication in human macrophages. Proc. Natl. Acad. Sci. U.S.A. 89, 9607-9611. doi: 10.1073/pnas.89.20.9607

McDade, J. E., Shepard, C. C., Fraser, D. W., Tsai, T. R., Redus, M. A., and Dowdle, W. R. (1977). Legionnaires' disease: isolation of a bacterium and demonstration of its role in other respiratory disease. N. Engl. J. Med. 297, 1197-1203. doi: 10.1056/NEJM197712012972202

Molmeret, M., Zink, S. D., Han, L., Abu-Zant, A., Asari, R., Bitar, D. M., et al. (2004). Activation of caspase-3 by the Dot/Icm virulence system is essential for arrested biogenesis of the Legionella-containing phagosome. Cell Microbiol. 6, 33-48. doi: 10.1046/j.1462-5822.2003. 00335.x

Mulazimoglu, L., and Yu, V. L. (2001). Can Legionnaires disease be diagnosed by clinical criteria? A critical review. Chest 120, 1049-1053.

Nogueira, C. V., Lindsten, T., Jamieson, A. M., Case, C. L., Shin, S., Thompson, C. B., et al. (2009). Rapid pathogen-induced apoptosis: a mechanism used by dendritic cells to limit intracellular replication of Legionella pneumophila. PLoS Pathog. 5:e1000478. doi: 10.1371/journal.ppat. 1000478

Ohtsuka, S., Ishii, Y., Matsuyama, M., Ano, S., Morishima, Y., Yanagawa, T., et al. (2014). SQSTM1/p62/A170 regulates the severity of Legionella pneumophila pneumonia by modulating inflammasome activity. Eur. J. Immunol. 44, 10841092. doi: 10.1002/eji.201344091

Pereira, M. S., Marques, G. G., Dellama, J. E., and Zamboni, D. S. (2011a). The Nlrc4 Inflammasome Contributes to Restriction of Pulmonary Infection by Flagellated Legionella spp. that Trigger Pyroptosis. Front. Microbiol. 2:33. doi: 10.3389/fmicb.2011.00033

Pereira, M. S., Morgantetti, G. F., Massis, L. M., Horta, C. V., Hori, J. I., and Zamboni, D. S. (2011b). Activation of NLRC4 by flagellated bacteria triggers caspase-1-dependent and -independent responses to restrict Legionella pneumophila replication in macrophages and in vivo. J. Immunol. 187, 64476455. doi: 10.4049/jimmunol.1003784

Pilla, D. M., Hagar, J. A., Haldar, A. K., Mason, A. K., Degrandi, D., Pfeffer, K., et al. (2014). Guanylate binding proteins promote caspase-11-dependent pyroptosis in response to cytoplasmic LPS. Proc. Natl. Acad. Sci. U.S.A. 111, 6046-6051. doi: $10.1073 /$ pnas.1321700111

Ren, T., Zamboni, D. S., Roy, C. R., Dietrich, W. F., and Vance, R. E. (2006). Flagellin-deficient Legionella mutants evade caspase-1- and Naip5-mediated macrophage immunity. PLoS Pathog. 2:e18. doi: 10.1371/journal.ppat.0020018

Richards, A. M., Von Dwingelo, J. E., Price, C. T., and Abu Kwaik, Y. (2013). Cellular microbiology and molecular ecology of Legionella-amoeba interaction. Virulence 4, 307-314. doi: 10.4161/viru.24290

Rossier, O., Starkenburg, S. R., and Cianciotto, N. P. (2004). Legionella pneumophila type II protein secretion promotes virulence in the $\mathrm{A} / \mathrm{J}$ mouse model of Legionnaires' disease pneumonia. Infect. Immun. 72, 310-321. doi: 10.1128/IAI.72.1.310-321.2004

Roy, C. R., Berger, K. H., and Isberg, R. R. (1998). Legionella pneumophila DotA protein is required for early phagosome trafficking decisions that occur within minutes of bacterial uptake. Mol. Microbiol. 28, 663-674. doi: 10.1046/j.13652958.1998.00841.x

Samrakandi, M. M., Cirillo, S. L., Ridenour, D. A., Bermudez, L. E., and Cirillo, J. D. (2002). Genetic and phenotypic differences between Legionella pneumophila strains. J. Clin. Microbiol. 40, 1352-1362. doi: 10.1128/JCM.40.4.13521362.2002
Santic, M., Asare, R., Doric, M., and Abu Kwaik, Y. (2007). Host-dependent trigger of caspases and apoptosis by Legionella pneumophila. Infect. Immun. 75, 2903-2913. doi: 10.1128/IAI.00147-07

Schroeder, G. N., Petty, N. K., Mousnier, A., Harding, C. R., Vogrin, A. J., Wee, B., et al. (2010). Legionella pneumophila strain 130b possesses a unique combination of type IV secretion systems and novel Dot/Icm secretion system effector proteins. J. Bacteriol. 192, 6001-6016. doi: 10.1128/JB. 00778-10

Shi, J., Zhao, Y., Wang, Y., Gao, W., Ding, J., Li, P., et al. (2014). Inflammatory caspases are innate immune receptors for intracellular LPS. Nature 514, 187192. doi: $10.1038 /$ nature 13683

Silveira, T. N., and Zamboni, D. S. (2010). Pore formation triggered by Legionella spp. is an Nlrc4 inflammasome-dependent host cell response that precedes pyroptosis. Infect. Immun. 78, 1403-1413. doi: 10.1128/IAI. 00905-09

Sun, G. W., Lu, J., Pervaiz, S., Cao, W. P., and Gan, Y. H. (2005). Caspase-1 dependent macrophage death induced by Burkholderia pseudomallei. Cell Microbiol. 7, 1447-1458. doi: 10.1111/j.1462-5822.2005. 00569.x

Tai, J., Benchekroun, M. N., Ennaji, M. M., Mekkour, M., and Cohen, N. (2012). Nosocomial Legionnaires' Disease: risque and prevention. Front. Sci. 2:75. doi: $10.5923 /$ j.fs. 20120204.03

Tao, L., Zhu, W., Hu, B. J., Qu, J. M., and Luo, Z. Q. (2013). Induction of rapid cell death by an environmental isolate of Legionella pneumophila in mouse macrophages. Infect. Immun. 81, 3077-3088. doi: 10.1128/IAI.00252-13

Tateda, K., Moore, T. A., Deng, J. C., Newstead, M. W., Zeng, X., Matsukawa, A., et al. (2001a). Early recruitment of neutrophils determines subsequent T1/T2 host responses in a murine model of Legionella pneumophila pneumonia. J. Immunol. 166, 3355-3361. doi: 10.4049/jimmunol.166.5.3355

Tateda, K., Moore, T. A., Newstead, M. W., Tsai, W. C., Zeng, X., Deng, J. C., et al. (2001b). Chemokine-dependent neutrophil recruitment in a murine model of Legionella pneumonia: potential role of neutrophils as immunoregulatory cells. Infect. Immun. 69, 2017-2024. doi: 10.1128/IAI.69.4.2017-2024. 2001

Tilney, L. G., Harb, O. S., Connelly, P. S., Robinson, C. G., and Roy, C. R. (2001). How the parasitic bacterium Legionella pneumophila modifies its phagosome and transforms it into rough ER: implications for conversion of plasma membrane to the ER membrane. J. Cell Sci. 114, $4637-4650$.

Tsuji, N. M., Tsutsui, H., Seki, E., Kuida, K., Okamura, H., Nakanishi, K., et al. (2004). Roles of caspase-1 in Listeria infection in mice. Int. Immunol. 16, 335-343. doi: 10.1093/intimm/dxh041

Vinzing, M., Eitel, J., Lippmann, J., Hocke, A. C., Zahlten, J., Slevogt, H., et al. (2008). NAIP and Ipaf control Legionella pneumophila replication in human cells. J. Immunol. 180, 6808-6815. doi: 10.4049/jimmunol.180.10.6808

von Moltke, J., Trinidad, N. J., Moayeri, M., Kintzer, A. F., Wang, S. B., Van Rooijen, N., et al. (2012). Rapid induction of inflammatory lipid mediators by the inflammasome in vivo. Nature 490, 107-111. doi: 10.1038/nature11351

Wright, E. K., Goodart, S. A., Growney, J. D., Hadinoto, V., Endrizzi, M. G., Long, E. M., et al. (2003). Naip5 affects host susceptibility to the intracellular pathogen Legionella pneumophila. Curr. Biol. 13, 27-36. doi: 10.1016/S09609822(02)01359-3

Yamamoto, Y., Klein, T. W., Newton, C. A., and Friedman, H. (1988). Interaction of Legionella pneumophila with peritoneal macrophages from various mouse strains. Adv. Exp. Med. Biol. 239, 89-98. doi: 10.1007/978-1-4757-5421-6_10

Yamamoto, Y., Klein, T. W., Newton, C. A., Widen, R., and Friedman, H. (1987). Differential growth of Legionella pneumophila in guinea pig versus mouse macrophage cultures. Infect. Immun. 55, 1369-1374.

Yoshida, S., Goto, Y., Mizuguchi, Y., Nomoto, K., and Skamene, E. (1991). Genetic control of natural resistance in mouse macrophages regulating intracellular Legionella pneumophila multiplication in vitro. Infect. Immun. 59, 428-432.

Zamboni, D. S., Kobayashi, K. S., Kohlsdorf, T., Ogura, Y., Long, E. M., Vance, R. E., et al. (2006). The Bircle cytosolic pattern-recognition receptor contributes to the detection and control of Legionella pneumophila infection. Nat. Immunol. 7 , 318-325. doi: 10.1038/ni1305

Zhao, Y., Yang, J., Shi, J., Gong, Y. N., Lu, Q., Xu, H., et al. (2011). The NLRC4 inflammasome receptors for bacterial flagellin and type III secretion apparatus. Nature 477, 596-600. doi: 10.1038/nature10510 
Zhu, W., Banga, S., Tan, Y., Zheng, C., Stephenson, R., Gately, J., et al. (2011). Comprehensive identification of protein substrates of the Dot/Icm type IV transporter of Legionella pneumophila. PLOS ONE 6:e17638. doi: 10.1371/journal.pone.0017638

Zhu, W., Hammad, L. A., Hsu, F., Mao, Y., and Luo, Z. Q. (2013). Induction of caspase 3 activation by multiple Legionella pneumophila Dot/Icm substrates. Cell Microbiol. 15, 1783-1795. doi: 10.1111/cmi.12157

Zink, S. D., Pedersen, L., Cianciotto, N. P., and Abu-Kwaik, Y. (2002). The Dot/Icm type IV secretion system of Legionella pneumophila is essential for the induction of apoptosis in human macrophages. Infect Immun. 70, 1657-1663. doi: 10.1128/IAI.70.3.1657-1663.2002
Conflict of Interest Statement: The authors declare that the research was conducted in the absence of any commercial or financial relationships that could be construed as a potential conflict of interest.

Copyright (c) 2016 Krause and Amer. This is an open-access article distributed under the terms of the Creative Commons Attribution License (CC BY). The use, distribution or reproduction in other forums is permitted, provided the original author(s) or licensor are credited and that the original publication in this journal is cited, in accordance with accepted academic practice. No use, distribution or reproduction is permitted which does not comply with these terms. 